\title{
The Role of microRNAs in Colorectal Cancer
}

\author{
Aaron J. Schetter, Hirokazu Okayama, and Curtis C. Harris \\ Laboratory of Human Carcinogenesis, Center for Cancer Research, National Cancer Institute, \\ National Institutes of Health, Bethesda, Maryland 20892, USA
}

\begin{abstract}
Over the last decade, it has become clear that aberrant microRNA expression has a functional role in the initiation and progression of colorectal cancer (CRC). Specific microRNAs can act as either tumor suppressors or oncogenes depending on the cellular environment in which they are expressed. The expression of microRNAs is reproducibly altered in CRC and their expression patterns are associated with diagnosis, prognosis and therapeutic outcome in CRC. Studies have begun to examine the association of microRNA related polymorphisms and their association with CRC incidence and prognosis as well as the possibility of using circulating microRNAs or fecal microRNA expression as non-invasive early detection biomarkers. These data suggest that microRNAs may be potential molecular classifiers, early detection biomarkers and therapeutic targets for CRC. Here, we will review the evidence demonstrating a role of microRNAs in CRC.
\end{abstract}

\section{Introduction}

Colorectal cancer (CRC) is responsible for $10 \%$ of the world-wide cancer incidence and mortality ${ }^{1}$; thus it represents a significant health burden. The more we understand about the etiological and biological nature of $\mathrm{CRC}$, the better equipped we will be at designing effective preventive, diagnostic and therapeutic tools to help reduce the burden of this disease. Practical issues that are being addressed include identifying effective therapeutic strategies, identifying the precise patient populations that may benefit from adjuvant chemotherapy and identifying precise patient populations that would benefit from intensive screening for disease recurrence. For example, TNM staging is arguably the best prognostic classier tool that helps guide therapeutic decisions for CRC ${ }^{2}$. Adjuvant therapy after surgical resection is considered to be beneficial for TNM stage III patients, yet it is controversial if adjuvant therapy should be given to TNM stage II patients ${ }^{3,4}$. Therefore there is a need to develop better classifiers to identify subpopulations of TNM stage II patients who would benefit from therapy.

Sporadic colon cancer progresses from a benign polyp to a malignant adenocarcinoma while cells accumulate a series of well documented genetic and epigenetic changes. Specific

Corresponding Author: Curtis C. Harris, MD, Laboratory of Human Carcinogenesis, Center for Cancer Research, NCI, NIH, 37 Convent Drive, Room 3068A, MSC 4258, Bethesda, MD 20892-4258, Phone 301/496-2048, curtis_harris@ @nih.gov.

Aaron J. Schetter, Laboratory of Human Carcinogenesis, Center for Cancer Research, NCI, NIH, 37 Convent Drive, Rm 3068A, MSC 4258, Bethesda, MD 20892-4258, Phone 301/496-7251, schettera@ mail.nih.gov

Hirokazu Okayama, Laboratory of Human Carcinogenesis, Center for Cancer Research, NCI, NIH, 37 Convent Drive, Building 37, Room3060, Bethesda, Maryland 20892, Phone 301/496-7252, okayamah@ mail.nih.gov

Publisher's Disclaimer: This is a PDF file of an unedited manuscript that has been accepted for publication. As a service to our customers we are providing this early version of the manuscript. The manuscript will undergo copyediting, typesetting, and review of the resulting proof before it is published in its final citable form. Please note that during the production process errors may be discovered which could affect the content, and all legal disclaimers that apply to the journal pertain.

Conflicts of Interest and Source of Funding: All authors receive funding from Intramural Research Program of the National Cancer Institute, National Institutes of Health. 
genetic changes are starting to inform therapeutic approches and guide treatment decisions. For example, both vascular endothelial growth factor (VEGF) and epidermal growth factor (EGFR) pathways have been shown to be critical for colon cancer progression. Targeted therapies to both EGFR and VEGF have shown promise in late stage CRC. ${ }^{5}$ KRAS is an important oncogene in CRC and mutations in KRAS predict a failure to respond to EGFR inhibitors. As we continue improve our understanding of the biology and both genetic and epigenetic changes in CRC, we may be able to develop additional biomarkers and therapies to help treat and even prevent this disease.

Historically, most research on CRC has focused on genetic and epigenetic changes in protein coding genes for their role in CRC initiation and progression. Recently, a class of small non-coding RNAs, called microRNAs, has come into focus. MicroRNAs are small, 18-24 nucleotide RNAs that regulate the translation and stability of specific target mRNAs. Ten years ago, microRNAs, were implicated in the initiation of chronic lymphocytic leukemia. ${ }^{6}$ Since that discovery, microRNAs have been shown to be involved in almost every aspect of cancer biology, as tumor suppressor genes or oncogenes ostensibly depending on the cellular context in which they are expressed. Evidence supports a role for microRNAs at every stage of CRC initiation, progression and development. Extensive research is now aimed at determining if microRNAs can be used as diagnostic biomarkers and therapeutic targets for cancer (Figure 1).

In this review, we will focus on the role of microRNAs in CRC and discuss their potential as diagnostic and prognostic classifiers for CRC and as therapeutic targets.

\section{MicroRNA expression is consistently altered in CRC}

To date, more than twenty studies have examined microRNA expression patterns in CRC and confirmed that microRNAs are consistently and reproducibly altered in this disease. ${ }^{7}$ These studies used a variety of techniques ranging from global miRNA expression profiling with deep sequencing ${ }^{8}$ or microRNA microarrays ${ }^{9-11}$ to examining the expression of selected microRNAs with quantitative reverse transcriptase polymerase chain reaction (qRTPCR). An underlying theme of all of these studies is that microRNA expression of CRC is distinctly different than nontumor tissues, which is consistent with the hypothesis that aberrant microRNA expression has a role in the CRC initiation and development.

In contrast to the original report that microRNA expression levels are globally reduced in cancer, ${ }^{11}$ more microRNAs have been found to have elevated expression in CRC compared to those with reduced levels. A recent review of 23 microRNA expression studies found that of the 164 microRNAs that are significantly altered in CRC in at least one study, approximately $2 / 3$ of them were elevated and $1 / 3$ that were reduced in tumors. ${ }^{7}$ This indicates that microRNAs may have more oncogenic than tumor suppressive functions in CRC. It also suggests that the microRNA processing machinery is not compromised in CRC. The number of microRNAs showing elevated expression in CRC corresponds with the findings that microRNAs are over-represented at regions of the genome that show copynumber gain while they are under-represented in regions that show copy number loss in CRC. ${ }^{12,13}$ Regardless of these findings, it is clear from functional studies that certain microRNAs have important oncogenic functions while others have important tumor suppressor functions and these functions need to be evaluated for each microRNA individually in the context of the specific tissue/tumor type.

Michael and colleagues were the first to show that microRNA expression patterns were altered in CRC. ${ }^{14}$ They reported that miR-143 and miR-145 were reduced in CRC and suggested that these microRNAs were tumor suppressors. Multiple studies have since validated these findings and demonstrated that miR-143 and miR-145 indeed have tumor 
suppressive functions in CRC. ${ }^{15}$ Another highly relevant microRNA in CRC is the oncogenic microRNA, miR-21. At least seven studies reported that miR-21 is elevated in CRC. ${ }^{7}$ Furthermore, miR-21 has been found to be elevated in many other solid tumor types ${ }^{10}$ and this microRNA has important roles in cancer initiation, progression and metastasis. Other microRNAs which have been found to be altered in CRC in multiple reports include the miR-17-92 cluster, miR-106a, miR-31, miR-181b, miR-183, miR-135a/b, the $\mathrm{miR}-200 \mathrm{a} / \mathrm{b} / \mathrm{c}$ family, miR-203 and miR-224. ${ }^{7}$

The transcriptional regulation of microRNAs is as complex and diverse as the transcriptional regulation of protein coding genes. Therefore, the causes of the altered expression of microRNAs in CRC are equally diverse and complex. Aberrant transcription of microRNAs in CRC can be the result of transcription factors that are activated through various oncogenic signaling cascades, the result of genomic amplification/loss, genotoxic stress or inflammatory stimuli. Epigenetic mechanisms also affect microRNA expression levels. Genome-wide profiling of chromatin signatures demonstrated that DNA methylation regulates miRNA expression of CRC cell lines in vitro. ${ }^{16}$ Several microRNAs, including let-7, ${ }^{17} \mathrm{miR}-34,{ }^{18} \mathrm{miR}-342,{ }^{19} \mathrm{miR}-345,{ }^{19} \mathrm{miR}-9,{ }^{20} \mathrm{miR}-129,{ }^{20}$ and miR- $137{ }^{20}$ are frequently hypermethylated in colon tumors and this is thought to lead to their reduced expression. MicroRNAs can also contribute to global epigenetic regulation in CRC. For example, miR-143 is a tumor suppressor microRNA that directly targets DNA methyltransferase 3A (DNMT3A) and loss of miR-143 expression leads to increased DNMT3A in CRC tissues. ${ }^{21}$ Similarly, loss of miR-342 leads to increased DNA methyltransferase 1 (DNMT1) and this contributes to the hypermethylation of several tumor suppressor genes in CRC. ${ }^{22}$

MicroRNA expression patterns can also classify tissue types and tumor types and microRNA expression patterns perform at least as well as mRNA expression profiles for this purpose. ${ }^{10,11}$ Therefore, microRNA expression pattern may help classify different phenotypic subgroups of CRC. Important phenotypic subgroups of CRC include microsatellite instability (MSI), KRAS mutation status, and TP53 status. These subgroups differ in therapeutic response and, thus, projected survival. MicroRNA expression patterns are different between microsatellite stable (MSS) and MSI tumors ${ }^{23-25}$ and these expression patterns can be used to correctly classify MSS versus MSI tumors. Oncogenic miR-155 targets the DNA mismatch repair proteins MLH1, MSH2, and MSH6 to contribute to the MSI phenotype in CRC. ${ }^{26}$ MiR-21 also targets MSH2, which is thought to increase tumor resistance to 5 fluorouracil based therapies. ${ }^{27}$ KRAS mutant tumors also show altered microRNA expression patterns when compared to wild type tumor. ${ }^{28}$ These findings demonstrate the potential for microRNA expression patterns to classify CRC tumors into different phenotypic groups. Altered microRNA expression may also explain some of the biological differences between each subgroup.

\section{MicroRNAs function at early stages in CRC development}

Adenomas are benign growths that are frequently a precursor lesion of colon adenocarcinoma. If microRNAs are altered in adenomas, it suggests that microRNAs have a role in the initiation of cancer. This is indeed the case. MicroRNA expression patterns can distinguish normal colonic mucosa, colon adenomas, and colon carcinomas. ${ }^{29}$ These expression patterns are consistent with the stepwise, multi-hit model for colon carcinogenesis and support a role for microRNAs in each step. MiR-21 is a good example of this in that it is elevated in both adenomas and colon carcinomas. ${ }^{9}$ Higher expression levels of miR-21 correlate with more advanced stages of CRC indicating a potential a role for miR-21 in the initiation and progression of CRC. ${ }^{9,}{ }^{30}$ Consistent with this, the frequency and extent of miR-21 expression by in situ hybridization was found to be increased during the 
transition from adenoma to advanced carcinoma. ${ }^{31}$ Elevated expression of miR-21 in mice can induce malignancies that are addicted to high miR-21 expression, demonstrating potential for this microRNA to initiate malignancies. ${ }^{32}$ MiR-135b may also have a role in the early stages of CRC. It is elevated in colon adenomas and one of its targets include the adenomatous polyposis coli (APC) gene, a component of the Wnt signaling pathway that is frequently mutated or repressed in adenomas. ${ }^{33}$ The miR-17-92 cluster of oncogenic microRNAs has increased expression in adenomas and thought to have a role the adenoma to carcinoma transition. ${ }^{34}$ Epigenetic silencing of miR- $137^{35}$ and miR-342 ${ }^{19}$ in colon adenomas also suggest a role for these microRNAs CRC.

Colon adenomas have high levels of cellular senescence that serve as a barrier to a malignant transformation ${ }^{36}$. MicroRNA expressions patterns may help maintain this barrier to prevent tumor progression. The $\mathrm{p} 53$ isoforms $\Delta 133 \mathrm{p} 53$ and $\mathrm{p} 53 \beta$ are endogenous regulators of cellular senescence and their expression patterns are consistent with preventing the progression from colon adenoma to carcinoma. ${ }^{37}$ MiR-34a is a p53 regulated microRNA that influences cellular senescence. ${ }^{37}$ The indirect repression of miR-34a by $\Delta 133 \mathrm{p} 53$ through the inhibition of p53 function may be one of the mechanisms that p53 isoforms utilize to bypass this senescence barrier.

\section{MicroRNA expression affects cell growth, proliferation, invasion and metastasis in CRC}

There is overwhelming evidence that supports a mechanistic role for specific microRNAs in CRC. Several microRNAs have been shown to have roles in CRC and their targets have been experimentally verified. While we cannot discuss all of those findings in this review, we will present some of the important findings on specific microRNAs, their targets, and their role colon cancer (Table 1).

The first study examining microRNA expression in colon tumors identified miR-143 and miR-145 as potential tumor suppressors. ${ }^{14}$ Since that time evidence has continued to support a tumor suppressive role for these microRNAs. Both miR-143 and miR-145 can regulate cell growth and proliferation in vitro, ${ }^{38}$ each by targeting different oncogenic protein coding genes. MiR-143 functions to suppress cell growth and proliferation by directly repressing the translation of KRAS,${ }^{39}$ DNMT3A $^{21}$ and extracellular signal-regulated kinase-5 (ERK5). ${ }^{38}$ Furthermore, replacement of miR-143 in xenograft mouse models of colon cancer reduced tumor growth ${ }^{15}$ supporting a functional role for miR-143 expression in colon cancer progression. The tumor suppressor activity of miR-145 functions in part through its inhibition of the oncogenic insulin receptor substrate-1 (IRS-1), ${ }^{40} \mathrm{c}-\mathrm{Myc},{ }^{41}$ Yamaguchi sarcoma viral oncogene homolog 1 (YES1), ${ }^{42}$ signal transducer and activator of transcription 1 (STAT1) ${ }^{42}$ and Friend leukemia integration 1 (FLI1) ${ }^{43}$ MiR-145 may also have a role in stem cell maintenance by targeting octamer-4 (OCT4), sex determining region Y-box 2 (SOX2) and Krüppel-like factor-4 (KLF4). ${ }^{44}$

MiR-21 is an important oncogenic microRNA with roles in tumor initiation, progression and metastasis. This microRNA is over expressed in at least 18 malignancies, indicating that miR-21 biology is relevant to most cancer types. ${ }^{10,45}$ Elevated miR-21 expression leads to increased cell proliferation, decreased apoptosis, increased cell migration, intravasation and metastasis by targeting several tumor suppressor genes. Tumor suppressor genes experimentally-verified to be targeted by miR-21 include programmed cell death 4 (PDCD4), ${ }^{46,47}$ phosphatase and tensin homolog (PTEN) ${ }^{48}$ Cell division cycle 25 homolog A (Cdc25a), ${ }^{49}$ reversion-inducing-cysteine-rich protein with kazal motifs (RECK), ${ }^{50}$ TIMP $3,{ }^{50}$ maspin, ${ }^{51}$ nuclear factor 1 B-type (NFIB), ${ }^{52}$ tropomyosin 1 (TPM1),${ }^{53}$ sprouty 2 (SPRY2), ${ }^{54} \mathrm{~T}$-lymphoma invasion and metastasis-inducing protein 1 (TIAM1), ${ }^{55}$ and Ras 
homolog gene family, member B (RHOB). ${ }^{56}$ The targeting of PDCD4 by miR-21 is of particular interest because it has been thoroughly validated in vitro, in mouse models, and the reverse association between miR-21 and PDCD4 protein expression has been confirmed in human tissues and appears to be relevant in multiple tumor types.

Several other microRNAs have been implicated in CRC. MiR-30a-5p is a tumor suppressor microRNA that targets denticleless homolog (DTL) to suppress tumor growth in CRC ${ }^{57}$. MiR-192 and miR-215 are both effectors and regulators of p53 function to suppress colon carcinogenesis. ${ }^{58}$ Another p53 related microRNA, miR-34a, has been shown inhibit cell invasion in colon cancer cell lines by targeting FRA $1 .{ }^{59}$ Cyclooxygenase $2(\mathrm{COX}-2)$ can be negatively regulated by miR-101 60 and this may contribute to the initiation and progression of colon tumors. MiR-451 overexpression in colon cancer cells leads to reduced cell proliferation through targeting of the oncogene macrophage migration factor (MIF) ${ }^{61}$ Over expression of miR-499-5p in CRC cell lines targets FOXO4 and PDCD4 to promote cell migration and invasion in vitro and lung and liver metastases in mouse models. ${ }^{62}$ MiR-675 can target the retinoblastoma (RB) tumor suppressor gene to increase tumor growth. ${ }^{63}$ MiR-365 acts as a tumor suppressor to inhibit cell cycle progression and promotes apoptosis of colon cancer cells by targeting Cyclin D1 (CCND1) and Bcl-2. ${ }^{64}$ Loss of miR-29 leads to increased expression of MMP2 to promote metastases in mouse models of colon cancer. ${ }^{65}$ The oncogenic miR-95 promotes tumorgenicity by targeting sorting nexin 1 (SNX1). ${ }^{66}$ Because altered microRNA expression can influence the initiation and progression of colon cancer, it suggests that microRNAs have potential as therapeutic targets for CRC.

\section{MicroRNA related polymorphisms and the risk and prognosis of CRC}

Recent studies suggest that $35 \%$ of all CRC can be attributed to inherited genetic factors. ${ }^{67}$ These inherited risks are likely due to single-nucleotide polymorphisms (SNPs) and other genetic abnormalities in both coding and noncoding genes. Because microRNAs have an important role in CRC initiation and development, it is possible that SNPs that disrupt microRNAs, microRNA binding sites, or microRNA processing may alter an individual's susceptibility to CRC. ${ }^{68}$ SNPs in the 3' untranslated region (UTR) of protein coding genes can also introduce microRNA binding sites to hasten translational repression of that gene. Therefore, microRNA related SNPs can deregulate biological pathways in a variety of ways and it is important to evaluate the association between these SNPs and CRC risk.

Studies evaluating the association between SNPs in microRNAs and microRNA binding sites with risk of CRC are starting to emerge. Zhan and colleagues reported that a SNP in miR-196a led to altered miR-196a expression and increased CRC incidence in a Chinese population consisting of $252 \mathrm{CRC}$ patients and 543 healthy controls. ${ }^{69} \mathrm{~A}$ global analysis of the 3'UTRs of candidate genes led to the identification of two SNPs; one which disrupts the binding of $m i R-337, m i R 582, m i R-200 a^{*}, m i R-184$ and $m i R-212$ for $C D 86$ and another which disrupts the binding of $m i R-612$ to INSR. In a study population of 697 cases and 624 controls, each of these SNPs were associated with cancer risk. ${ }^{70}$ Zanetti and colleagues have recently reported that a SNP disrupts the binding of miR-27a to the 3' UTR $M B L 2$ and this SNP was associated with colon cancer risk in an African American population of 100 cases and 186 controls while a similar association was not found in Caucasians. ${ }^{71}$ While the findings of each of these studies require validation in additional, independent populations and the biological mechanism that are responsible for these associations need to be further explored, these studies support the hypothesis that SNPs in microRNAs and microRNA binding sites modify colon cancer risk.

Similar studies are evaluating microRNA related SNPs and their association with prognosis and response to therapy. For example, a study of 426 CRC patients found that a SNP in 
miR-492 was modestly associated with improved survival ${ }^{72}$. KRAS-LCS6 is a particular SNP of interest as it disrupts a let-7 binding site in the 3'UTR of KRAS. CRC patients with activating KRAS mutations do not respond to anti-EGFR therapies. Because KRAS-LCS6 may lead to altered levels of KRAS protein, it leads to the hypothesis that KRAS-LCS6 may have some association with therapeutic response. In a study of 121 metastatic CRC patients without BRAF mutations who received anti-EGFR therapy, patients with KRAS-LCS6 showed significantly worse overall survival. ${ }^{73}$ Although a similar study evaluated 130 metastatic, KRAS wild type CRC patients and found that KRAS-LCS6 was associated with improved response to anti-EGFR therapy. ${ }^{74}$ In a third study of 409 early stage, 182 stage III, and 69 stage IV CRC cases, KRAS-LCS6 was associated with improved survival only in early stage cases and no survival benefit was observed in late stage patients. ${ }^{75}$ These seemingly contradictory results highlight the need for independent validation in multiple cohorts with special attention to KRAS and BRAF mutation status and their interaction with specific chemotherapeutic regimens.

\section{micoRNAs as diagnostic biomarkers for CRC}

Undoubtedly, early detection of CRC provides the best chance for successful treatment. While current chemotherapeutic regimens have significantly improved overall survival of CRC patients, surgery before metastatic spreading of the disease is considered the only curative form of treatment. The screening methods of colonoscopies and fecal occult blood tests (FOBT) have improved survival rates for CRC by detecting patients and earlier stages of cancer, but there is still much room for improvement and neither test is ideal. While colonoscopies are considered the best screening tool because it can also remove precancerous polyps during the procedure, they are both invasive and expensive, which leads to lower compliance rates. FOBT is less invasive, but also less sensitive and specific. New non-invasive, accurate biomarkers are needed to improve both the accuracy and screening rates for CRC. MicroRNAs are being evaluated for their potential in this area.

Circulating microRNAs can be detected in blood serum or plasma. $\mathrm{Ng}$ and colleagues were the first to report that circulating levels of microRNAs were different in blood plasma in CRC cases and controls. ${ }^{76}$ In a population of 90 cases and 50 controls, they found that miR-92 was expressed at higher levels in the plasma cases and could distinguish cases from healthy control patients with $70 \%$ specificity and $89 \%$ sensitivity. The expression of miR-92 was reduced following surgical removal of the tumor suggesting that circulating levels of microRNAs may be useful marker of disease recurrence. A second study of 120 cases and 29 controls validated these findings showing that plasma levels of miR-92 can discriminate CRC cases and controls with $65 \%$ sensitivity and $82 \%$ specificity. ${ }^{77}$ While this classifier may not be accurate enough on its own to serve as a screening marker, it may be developed along with additional markers to improve screening accuracy. A similar study found that circulating levels of miR-141 has been shown to be elevated in metastatic CRC and its expression is associated with poor prognosis, suggesting that this microRNA may be used in conjunction with carcinoembryonic antigen (CEA) to detect CRC with distant metastases ${ }^{78}$.

Measuring microRNAs in stool offers another non-invasive approach to detect CRC. One small study of 29 CRC cases and 8 healthy controls found that stool from CRC cases expressed higher levels of miR-21 and miR-106a ${ }^{79}$. A larger study of 197 CRC cases and 134 healthy controls investigated microRNA expression patterns of colonocytes isolated from feces and was able to demonstrate that microRNA expression patterns could distinguish cases from controls with $74 \%$ sensitivity and $79 \%$ specificity. ${ }^{80} \mathrm{~A}$ similar strategy found that microRNA methylation patterns from DNA isolated from stool may have promise as a screening tool for CRC. ${ }^{81}$ Hypermethylation pattern of miR-34b/c in stool samples could distinguish CRC cases from controls with $75 \%$ sensitivity and $84 \%$ 
specificity. Further tests are warranted to determine if microRNA expression or methylation patterns in stool can be utilized, alone or in combination with FOBT, as an effective screening strategy for CRC.

\section{microRNA expression as prognostic and predictive biomarkers}

Molecular classifiers can serve as prognostic and predictive tools to help stratify cancer patients into appropriate risk groups to aid physicians in making therapeutic decisions. These decisions can include whether or not to provide adjuvant chemotherapy or what types of therapy are appropriate. Expression patterns of microRNAs are associated with both prognosis and therapeutic outcomes in CRC; therefore they have potential as prognostic and predictive biomarkers.

Elevated expression of miR-21 has a robust and reproducible association with colon cancer prognosis. We first reported this association based on both microRNA microarray expression data on an American cohort of 84 colon cancer patients and by qRT-PCR in an additional cohort of 113 Chinese patients. ${ }^{9}$ In both cohorts, elevated miR-21 expression in tumors was associated with worse survival prognosis and therapeutic outcomes. These associations were independent of staging and other clinical characteristics demonstrating the potential of miR-21 as an independent prognostic and predictive biomarker. This association was also significant in TNM stage II patients, demonstrating that miR-21 expression may be a useful, early stage biomarker to identify subjects at high risk of cancer progression that have no evidence for metastasis or advanced disease. The association of elevated miR-21 expression and worse survival outcomes in CRC has now been validated in at least three additional studies (Figure 2A). These include studies of 156 Japanese CRC patients, ${ }^{82} 46$ CRC patients from the Czech Republic ${ }^{83}$ and 130 TNM stage II colon cancer patients from Denmark. ${ }^{84}$ The fact that these associations are found in diverse ethnic populations using a variety of methods to measure miR-21 argues that miR-21 expression is a robust prognostic classifier for CRC that is valid for most populations regardless of ethnicity. In fact, the association of elevated miR-21 and worse prognosis has now been reported in at least 9 additional cancer types including lung cancer, ${ }^{85,} 86$ breast cancer, ${ }^{87}$ pancreatic cancer, ${ }^{88}$ tongue, ${ }^{89}$ gastric cancer ${ }^{90}$, head and neck cancer, ${ }^{91}$ chronic lymphocytic leukemia, ${ }^{92}$ melanoma ${ }^{93}$ and astrocytomas ${ }^{94}$. These findings are consistent with the hypothesis that miR-21 expression is a prognostic classifier for many types of malignancies.

Additional studies have identified microRNA expression patterns that are associated with either prognosis or therapeutic outcome. The expression levels of miR-106b, ${ }^{95}$ miR-320, ${ }^{25}$ miR-498, ${ }^{25}$ miR-125b, ${ }^{96}$ miR-145, ${ }^{97}$ miR-185, ${ }^{98}$ miR-133b, ${ }^{98}$ miR-215, ${ }^{99}$ and miR-17 ${ }^{100}$ have each been reported to be associated with prognosis or therapeutic outcome in CRC in at least one study. Elevated expression of Dicer, an important gene encoding and RNA nuclease that is involved in microRNA processing, is associated with poor prognosis in CRC. ${ }^{101}$ Further validation of these associations is warranted and may reveal additional prognostic classifiers for CRC.

The combination of multiple, independent validated biomarkers may provide improved risk stratification of patients (Figure 2B). As an example of this, we have reported that the combination miR-21 expression data with an inflammatory gene classifier and demonstrated significantly improved risk stratification with cancer-specific mortality. ${ }^{102}$ The combination was also significant in TNM stage II colon cancer patients indicating that these may also be useful in identifying patients that are more likely to have undetectable micro-metastases. 


\section{Altered microRNA expression may contribute to chemoresistance}

We have shown that miR-21 expression is associated with therapeutic outcome to 5FUbased therapies in both American and Chinese cohorts. ${ }^{9}$ This association, in combination with the known oncogenic role for miR-21, suggests that increased miR-21 expression is in part responsible for the resistance to $5 \mathrm{FU}$. Elevated miR-21 induces resistance to $5 \mathrm{FU}$ in colon cancer cell lines by down regulating DNA repair protein MutS homolog 2 (MSH2). ${ }^{27}$ Exposure of colon cancer cells to 5FU leads to the increased miR-21 expression and this may be a response to genotoxic stress to help cells overcome the effects of 5FU. ${ }^{103}$ Additional in vitro data support roles for altered expression of miR-140, ${ }^{104} \mathrm{miR}-215,{ }^{105}$ miR-224 106 and miR-20a ${ }^{107}$ in developing chemoresistance. Further studies are warranted to determine if the expression of these microRNAs can predict response to chemotherapy and if those microRNAs can be used as therapeutic targets themselves.

\section{Potential for microRNAs a therapeutic target}

There is an emphasis to determine if microRNA-based therapies can be used to treat cancer. The two general strategies for microRNA-based therapies are to inhibit oncogenic microRNAs or restoring tumor suppressor microRNAs. Preclinical models have shown that both strategies can be effective.

Direct inhibition of microRNAs can be achieved by using antisense oligonucleotides or microRNA sponges to bind and sequester the target microRNA. Antagomirs are modified, antisense oligonucleotides which have been used to inhibit microRNAs in vivo ${ }^{108}$ in mouse studies. Similar strategies have been used to effectively inhibit miR-122 function in primates $^{109}$ and anti-miR-122 treatments in chimpanzees chronically infected with HCV can improve HCV-related liver pathology ${ }^{110}$. This anti-miR-122 drug has progressed to Phase II clinical trials to treat HCV in humans suggesting that anti-sense based microRNA therapeutics may soon be available. Indirect inhibition of microRNAs may be achieved through treatment with various chemical compounds. In fact, screens have found small molecule inhibitors that can inhibit the function of at least miR-21 ${ }^{111}$ and miR-122 112 in vitro.

MicroRNA replacement involves reintroducing synthetic microRNA mimics or expression vectors that will produce the microRNA of interest. This has shown promise in preclinical mouse models where reintroduction of miR-145 and miR-33a had an antitumor effect in a model of colon cancer. ${ }^{113}$

The critical issues to overcome with microRNA-based therapies is to have a delivery mechanism that allows prolonged stability of the therapy and provide sufficient uptake in target cells while minimizing off-target side effects. These strategies include optimizing the chemistry and size of the synthetic oligonucleotides to achieve better binding efficiency and increased stability. Delivery mechanisms range from the use adenoviral vector-based delivery, to cationic liposomes to polymer-based nanoparticles. There are great expectations that these strategies will result in effective therapies for CRC.

\section{Future Prospective and Conclusion}

MicroRNAs have a clear role in the initiation and progression of CRC. Future research will have to specifically address the potential role for microRNA-based classifiers and therapeutics in medicine.

Many studies have reported that microRNA expression patterns or microRNA related polymorphisms are associated with diagnosis or prognosis in CRC indicating that they have 
potential as early detection markers or predictive/prognostic classifiers. Like all populationbased studies, these too require validation in additional populations to determine if these results are representative of the majority of CRC. Most of these studies are retrospective analyses on historical cohorts; therefore, there is a need to conduct prospective studies asking how relevant the reported associations are to current CRC patients. We need to determine if microRNA classifiers can provide actionable information, such as how or even if to treat a patient. Assays measuring microRNAs have to be standardized in ways that will allow an assay to give clinically relevant information on a single patient.

The successful development of microRNA-based therapeutics for CRC has many challenging hurdles to overcome. First, the best microRNAs to be targeted in CRC are yet to be defined. In order to do this, a better understanding the biology of how these microRNAs affect CRC is required. Anti-miR-21 based therapies or microRNA replacement therapies for miR-143 and miR-145 may provide useful strategies based on what is already known about their biology. Another major obstacle is determining how to achieve effective delivery without causing unnecessary side effects. Finally, the appropriate patient populations have to be selected in order to determine if there strategies can have therapeutic benefit.

While there is still much to be done, we remain optimistic that microRNA related diagnostics and therapeutics have substantial potential for the prevention and treatment of CRC.

\section{Acknowledgments}

We thank Drs. Anna Robles and Brid Ryan for helpful discussions and critical review of this manuscript.

\section{References}

1. Jemal A, et al. Cancer statistics, 2010. CA Cancer J Clin. 2010; 60(5):277-300. [PubMed: 20610543]

2. Nagtegaal ID, Quirke P, Schmoll HJ. Has the new TNM classification for colorectal cancer improved care? Nat Rev Clin Oncol. 2011; 9(2):119-123. [PubMed: 22009076]

3. Chua YJ, Zalcberg JR. Progress and challenges in the adjuvant treatment of stage II and III colon cancers. Expert Rev Anticancer Ther. 2008; 8(4):595-604. [PubMed: 18402526]

4. Wolpin BM, Mayer RJ. Systemic treatment of colorectal cancer. Gastroenterology. 2008; 134(5): 1296-1310. [PubMed: 18471507]

5. Rousseau B, et al. Stage II and stage III colon cancer: treatment advances and future directions. Cancer J. 2010; 16(3):202-209. [PubMed: 20526097]

6. Calin GA, et al. Frequent deletions and down-regulation of micro- RNA genes miR15 and miR16 at 13q14 in chronic lymphocytic leukemia. Proc Natl Acad Sci U S A. 2002; 99(24):15524-15529. [PubMed: 12434020]

7. Luo X, et al. MicroRNA signatures: novel biomarker for colorectal cancer? Cancer Epidemiol Biomarkers Prev. 2011; 20(7):1272-1286. [PubMed: 21551242]

8. Cummins JM, et al. The colorectal microRNAome. Proc Natl Acad Sci U S A. 2006; 103(10):36873692. [PubMed: 16505370]

9. Schetter AJ, et al. MicroRNA expression profiles associated with prognosis and therapeutic outcome in colon adenocarcinoma. Jama. 2008; 299(4):425-436. [PubMed: 18230780]

10. Volinia S, et al. A microRNA expression signature of human solid tumors defines cancer gene targets. Proc Natl Acad Sci U S A. 2006; 103(7):2257-2261. [PubMed: 16461460]

11. Lu J, et al. MicroRNA expression profiles classify human cancers. Nature. 2005; 435(7043):834838. [PubMed: 15944708]

12. Lamy $\mathrm{P}$, et al. Are microRNAs located in genomic regions associated with cancer? Br J Cancer. 2006; 95(10):1415-1418. [PubMed: 17003783] 
13. Calin GA, et al. Human microRNA genes are frequently located at fragile sites and genomic regions involved in cancers. Proc Natl Acad Sci U S A. 2004; 101(9):2999-3004. [PubMed: 14973191]

14. Michael MZ, et al. Reduced accumulation of specific microRNAs in colorectal neoplasia. Mol Cancer Res. 2003; 1(12):882-891. [PubMed: 14573789]

15. Akao Y, et al. Role of anti-oncomirs miR-143 and-145 in human colorectal tumors. Cancer Gene Ther. 2010; 17(6):398-408. [PubMed: 20094072]

16. Suzuki H, et al. Genome-wide profiling of chromatin signatures reveals epigenetic regulation of MicroRNA genes in colorectal cancer. Cancer Res. 2011; 71(17):5646-5658. [PubMed: 21734013]

17. Brueckner B, et al. The human let-7a-3 locus contains an epigenetically regulated microRNA gene with oncogenic function. Cancer Res. 2007; 67(4):1419-1423. [PubMed: 17308078]

18. Toyota M, et al. Epigenetic silencing of microRNA-34b/c and B-cell translocation gene 4 is associated with $\mathrm{CpG}$ island methylation in colorectal cancer. Cancer Res. 2008; 68(11):41234132. [PubMed: 18519671]

19. Grady WM, et al. Epigenetic silencing of the intronic microRNA hsa-miR-342 and its host gene EVL in colorectal cancer. Oncogene. 2008; 27(27):3880-3888. [PubMed: 18264139]

20. Agirre $X$, et al. Epigenetic silencing of the tumor suppressor microRNA Hsa-miR-124a regulates CDK6 expression and confers a poor prognosis in acute lymphoblastic leukemia. Cancer Res. 2009; 69(10):4443-4453. [PubMed: 19435910]

21. Ng EK, et al. MicroRNA-143 targets DNA methyltransferases 3A in colorectal cancer. Br J Cancer. 2009

22. Wang H, et al. MicroRNA-342 inhibits colorectal cancer cell proliferation and invasion by directly targeting DNA methyltransferase 1. Carcinogenesis. 2011; 32(7):1033-1042. [PubMed: 21565830]

23. Lanza G, et al. mRNA/microRNA gene expression profile in microsatellite unstable colorectal cancer. Mol Cancer. 2007; 6:54. [PubMed: 17716371]

24. Sarver AL, et al. Human colon cancer profiles show differential microRNA expression depending on mismatch repair status and are characteristic of undifferentiated proliferative states. BMC Cancer. 2009; 9:401. [PubMed: 19922656]

25. Schepeler T, et al. Diagnostic and prognostic microRNAs in stage II colon cancer. Cancer Res. 2008; 68(15):6416-6424. [PubMed: 18676867]

26. Valeri N, et al. Modulation of mismatch repair and genomic stability by miR-155. Proc Natl Acad Sci U S A. 2010; 107(15):6982-6987. [PubMed: 20351277]

27. Valeri N, et al. MicroRNA-21 induces resistance to 5-fluorouracil by down-regulating human DNA MutS homolog 2 (hMSH2). Proc Natl Acad Sci U S A. 2010; 107(49):21098-21103. [PubMed: 21078976]

28. Mosakhani N, et al. MicroRNA profiling differentiates colorectal cancer according to KRAS status. Genes Chromosomes Cancer. 2012; 51(1):1-9. [PubMed: 21922590]

29. Oberg AL, et al. miRNA expression in colon polyps provides evidence for a multihit model of colon cancer. PLoS ONE. 2011; 6(6):e20465. [PubMed: 21694772]

30. Slaby O, et al. Altered expression of miR-21, miR-31, miR-143 and miR-145 is related to clinicopathologic features of colorectal cancer. Oncology. 2007; 72(5-6):397-402. [PubMed: 18196926]

31. Yamamichi N, et al. Locked nucleic acid in situ hybridization analysis of miR-21 expression during colorectal cancer development. Clin Cancer Res. 2009; 15(12):4009-4016. [PubMed: 19509156]

32. Medina PP, Nolde M, Slack FJ. OncomiR addiction in an in vivo model of microRNA-21-induced pre-B-cell lymphoma. Nature. 2010; 467(7311):86-90. [PubMed: 20693987]

33. Nagel R, et al. Regulation of the adenomatous polyposis coli gene by the miR-135 family in colorectal cancer. Cancer Res. 2008; 68(14):5795-5802. [PubMed: 18632633]

34. Diosdado B, et al. MiR-17-92 cluster is associated with $13 \mathrm{q}$ gain and c-myc expression during colorectal adenoma to adenocarcinoma progression. Br J Cancer. 2009; 101(4):707-714.

[PubMed: 19672269] 
35. Balaguer F, et al. Epigenetic silencing of miR-137 is an early event in colorectal carcinogenesis. Cancer Res. 2010; 70(16):6609-6618. [PubMed: 20682795]

36. Bartkova $\mathrm{J}$, et al. Oncogene-induced senescence is part of the tumorigenesis barrier imposed by DNA damage checkpoints. Nature. 2006; 444(7119):633-637. [PubMed: 17136093]

37. Fujita K, et al. p53 isoforms Delta133p53 and p53beta are endogenous regulators of replicative cellular senescence. Nat Cell Biol. 2009; 11(9):1135-1142. [PubMed: 19701195]

38. Akao Y, Nakagawa Y, Naoe T. MicroRNA-143 and-145 in colon cancer. DNA Cell Biol. 2007; 26(5):311-320. [PubMed: 17504027]

39. Chen X, et al. Role of miR-143 targeting KRAS in colorectal tumorigenesis. Oncogene. 2009; 28(10):1385-1392. [PubMed: 19137007]

40. Shi B, et al. Micro RNA 145 targets the insulin receptor substrate-1 and inhibits the growth of colon cancer cells. J Biol Chem. 2007; 282(45):32582-32590. [PubMed: 17827156]

41. Sachdeva M, et al. p53 represses c-Myc through induction of the tumor suppressor miR-145. Proc Natl Acad Sci U S A. 2009; 106(9):3207-3212. [PubMed: 19202062]

42. Gregersen LH, et al. MicroRNA-145 targets YES and STAT1 in colon cancer cells. PLoS ONE. 2010; 5(1):e8836. [PubMed: 20098684]

43. Zhang J, et al. Putative tumor suppressor miR-145 inhibits colon cancer cell growth by targeting oncogene Friend leukemia virus integration 1 gene. Cancer. 2011; 117(1):86-95. [PubMed: 20737575]

44. Xu N, et al. MicroRNA-145 regulates OCT4, SOX2, and KLF4 and represses pluripotency in human embryonic stem cells. Cell. 2009; 137(4):647-658. [PubMed: 19409607]

45. Selcuklu SD, Donoghue MT, Spillane C. miR-21 as a key regulator of oncogenic processes. Biochem Soc Trans. 2009; 37(Pt 4):918-925. [PubMed: 19614619]

46. Asangani IA, et al. MicroRNA-21 (miR-21) post-transcriptionally downregulates tumor suppressor Pdcd4 and stimulates invasion, intravasation and metastasis in colorectal cancer. Oncogene. 2008; 27(15):2128-2136. [PubMed: 17968323]

47. Lu Z, et al. MicroRNA-21 promotes cell transformation by targeting the programmed cell death 4 gene. Oncogene. 2008; 27(31):4373-4379. [PubMed: 18372920]

48. Meng F, et al. MicroRNA-21 regulates expression of the PTEN tumor suppressor gene in human hepatocellular cancer. Gastroenterology. 2007; 133(2):647-658. [PubMed: 17681183]

49. Wang P, et al. microRNA-21 negatively regulates Cdc25A and cell cycle progression in colon cancer cells. Cancer Res. 2009; 69(20):8157-8165. [PubMed: 19826040]

50. Gabriely G, et al. MicroRNA 21 promotes glioma invasion by targeting matrix metalloproteinase regulators. Mol Cell Biol. 2008; 28(17):5369-5380. [PubMed: 18591254]

51. Zhu S, et al. MicroRNA-21 targets tumor suppressor genes in invasion and metastasis. Cell Res. 2008; 18(3):350-359. [PubMed: 18270520]

52. Fujita $\mathrm{S}$, et al. miR-21 Gene expression triggered by AP-1 is sustained through a double-negative feedback mechanism. J Mol Biol. 2008; 378(3):492-504. [PubMed: 18384814]

53. Zhu S, et al. MicroRNA-21 Targets the Tumor Suppressor Gene Tropomyosin 1 (TPM1). J Biol Chem. 2007; 282(19):14328-14336. [PubMed: 17363372]

54. Sayed D, et al. MicroRNA-21 targets Sprouty2 and promotes cellular outgrowths. Mol Biol Cell. 2008; 19(8):3272-3282. [PubMed: 18508928]

55. Cottonham CL, Kaneko S, Xu L. miR-21 and miR-31 converge on TIAM1 to regulate migration and invasion of colon carcinoma cells. J Biol Chem. 2010; 285(46):35293-35302. [PubMed: 20826792]

56. Liu M, et al. miR-21 targets the tumor suppressor RhoB and regulates proliferation, invasion and apoptosis in colorectal cancer cells. FEBS Lett. 2011; 585(19):2998-3005. [PubMed: 21872591]

57. Baraniskin A, et al. MiR-30a-5p suppresses tumor growth in colon carcinoma by targeting DTL. Carcinogenesis. 2012

58. Braun CJ, et al. p53-Responsive micrornas 192 and 215 are capable of inducing cell cycle arrest. Cancer Res. 2008; 68(24):10094-10104. [PubMed: 19074875]

59. Wu J, et al. MicroRNA-34a inhibits migration and invasion of colon cancer cells via targeting to Fra-1. Carcinogenesis. 2012 
60. Strillacci A, et al. MiR-101 downregulation is involved in cyclooxygenase-2 overexpression in human colon cancer cells. Exp Cell Res. 2009; 315(8):1439-1447. [PubMed: 19133256]

61. Bandres E, et al. microRNA-451 regulates macrophage migration inhibitory factor production and proliferation of gastrointestinal cancer cells. Clin Cancer Res. 2009; 15(7):2281-2290. [PubMed: 19318487]

62. Liu X, et al. MicroRNA-499-5p promotes cellular invasion and tumor metastasis in colorectal cancer by targeting FOXO4 and PDCD4. Carcinogenesis. 2011; 32(12):1798-1805. [PubMed: 21934092]

63. Tsang WP, et al. Oncofetal H19-derived miR-675 regulates tumor suppressor RB in human colorectal cancer. Carcinogenesis. 2010; 31(3):350-358. [PubMed: 19926638]

64. Nie J, et al. microRNA-365, down-regulated in colon cancer, inhibits cell cycle progression and promotes apoptosis of colon cancer cells by probably targeting Cyclin D1 and Bcl-2. Carcinogenesis. 2012; 33(1):220-225. [PubMed: 22072615]

65. Ding Q, et al. APOBEC3G promotes liver metastasis in an orthotopic mouse model of colorectal cancer and predicts human hepatic metastasis. J Clin Invest. 2011; 121(11):4526-4536. [PubMed: 21985787]

66. Huang Z, et al. MicroRNA-95 promotes cell proliferation and targets sorting Nexin 1 in human colorectal carcinoma. Cancer Res. 2011; 71(7):2582-2589. [PubMed: 21427358]

67. Lichtenstein P, et al. Environmental and heritable factors in the causation of cancer--analyses of cohorts of twins from Sweden, Denmark, and Finland. N Engl J Med. 2000; 343(2):78-85. [PubMed: 10891514]

68. Ryan BM, Robles AI, Harris CC. Genetic variation in microRNA networks: the implications for cancer research. Nat Rev Cancer. 2010; 10(6):389-402. [PubMed: 20495573]

69. Zhan JF, et al. A functional variant in microRNA-196a2 is associated with susceptibility of colorectal cancer in a Chinese population. Arch Med Res. 2011; 42(2):144-148. [PubMed: 21565628]

70. Landi D, et al. Polymorphisms within micro-RNA-binding sites and risk of sporadic colorectal cancer. Carcinogenesis. 2008; 29(3):579-584. [PubMed: 18192692]

71. Zanetti KA, et al. 3'-UTR and Functional Secretor Haplotypes in Mannose-Binding Lectin 2 Are Associated with Increased Colon Cancer Risk in African Americans. Cancer Res. 2012

72. Lee HC, et al. Prognostic impact of microRNA-related gene polymorphisms on survival of patients with colorectal cancer. J Cancer Res Clin Oncol. 2010; 136(7):1073-1078. [PubMed: 20044760]

73. Graziano F, et al. Genetic modulation of the Let-7 microRNA binding to KRAS 3'-untranslated region and survival of metastatic colorectal cancer patients treated with salvage cetuximabirinotecan. Pharmacogenomics J. 2010; 10(5):458-464. [PubMed: 20177422]

74. Zhang W, et al. A let-7 microRNA-binding site polymorphism in 3'-untranslated region of KRAS gene predicts response in wild-type KRAS patients with metastatic colorectal cancer treated with cetuximab monotherapy. Ann Oncol. 2011; 22(1):104-109. [PubMed: 20603437]

75. Smits KM, et al. A let-7 microRNA SNP in the KRAS 3'UTR is prognostic in early-stage colorectal cancer. Clin Cancer Res. 2011; 17(24):7723-7731. [PubMed: 21994416]

76. $\mathrm{Ng}$ EK, et al. Differential expression of microRNAs in plasma of patients with colorectal cancer: a potential marker for colorectal cancer screening. Gut. 2009; 58(10):1375-1381. [PubMed: 19201770]

77. Huang Z, et al. Plasma microRNAs are promising novel biomarkers for early detection of colorectal cancer. Int J Cancer. 2010; 127(1):118-126. [PubMed: 19876917]

78. Cheng H, et al. Circulating plasma MiR-141 is a novel biomarker for metastatic colon cancer and predicts poor prognosis. PLoS ONE. 2011; 6(3):e17745. [PubMed: 21445232]

79. Link A, et al. Fecal MicroRNAs as novel biomarkers for colon cancer screening. Cancer Epidemiol Biomarkers Prev. 2010; 19(7):1766-1774. [PubMed: 20551304]

80. Koga Y, et al. MicroRNA expression profiling of exfoliated colonocytes isolated from feces for colorectal cancer screening. Cancer Prev Res (Phila). 2010; 3(11):1435-1442. [PubMed: 20959518]

81. Kalimutho M, et al. Epigenetically silenced miR-34b/c as a novel faecal-based screening marker for colorectal cancer. Br J Cancer. 2011; 104(11):1770-1778. [PubMed: 21610744] 
82. Shibuya $\mathrm{H}$, et al. Clinicopathological and prognostic value of microRNA-21 and microRNA-155 in colorectal cancer. Oncology. 2010; 79(3-4):313-320. [PubMed: 21412018]

83. Kulda V, et al. Relevance of miR-21 and miR-143 expression in tissue samples of colorectal carcinoma and its liver metastases. Cancer Genet Cytogenet. 2010; 200(2):154-160. [PubMed: 20620599]

84. Nielsen BS, et al. High levels of microRNA-21 in the stroma of colorectal cancers predict short disease-free survival in stage II colon cancer patients. Clin Exp Metastasis. 2011; 28(1):27-38. [PubMed: 21069438]

85. Markou A, et al. Prognostic value of mature microRNA-21 and microRNA-205 overexpression in non-small cell lung cancer by quantitative real-time RT-PCR. Clin Chem. 2008; 54(10):16961704. [PubMed: 18719201]

86. Yanaihara N, et al. Unique microRNA molecular profiles in lung cancer diagnosis and prognosis. Cancer Cell. 2006; 9(3):189-198. [PubMed: 16530703]

87. Yan LX, et al. MicroRNA miR-21 overexpression in human breast cancer is associated with advanced clinical stage, lymph node metastasis and patient poor prognosis. Rna. 2008; 14(11): 2348-2360. [PubMed: 18812439]

88. Dillhoff M, et al. MicroRNA-21 is overexpressed in pancreatic cancer and a potential predictor of survival. J Gastrointest Surg. 2008; 12(12):2171-2176. [PubMed: 18642050]

89. Li J, et al. MiR-21 indicates poor prognosis in tongue squamous cell carcinomas as an apoptosis inhibitor. Clin Cancer Res. 2009; 15(12):3998-4008. [PubMed: 19509158]

90. Jiang J, et al. Prognostic significance of miR-181b and miR-21 in gastric cancer patients treated with S-1/Oxaliplatin or Doxifluridine/Oxaliplatin. PLoS ONE. 2011; 6(8):e23271. [PubMed: 21876743]

91. Avissar M, et al. MicroRNA expression in head and neck cancer associates with alcohol consumption and survival. Carcinogenesis. 2009; 30(12):2059-2063. [PubMed: 19901002]

92. Rossi S, et al. microRNA fingerprinting of CLL patients with chromosome 17p deletion identify a miR-21 score that stratifies early survival. Blood. 2010; 116(6):945-952. [PubMed: 20393129]

93. Jiang L, et al. The status of microRNA-21 expression and its clinical significance in human cutaneous malignant melanoma. Acta Histochem. 2011

94. Zhi F, et al. The use of hsa-miR-21, hsa-miR-181b and hsa-miR-106a as prognostic indicators of astrocytoma. Eur J Cancer. 2010; 46(9):1640-1649. [PubMed: 20219352]

95. Diaz R, et al. Deregulated expression of miR-106a predicts survival in human colon cancer patients. Genes Chromosomes Cancer. 2008; 47(9):794-802. [PubMed: 18521848]

96. Nishida N, et al. MicroRNA miR-125b is a prognostic marker in human colorectal cancer. Int $\mathbf{J}$ Oncol. 2011; 38(5):1437-1443. [PubMed: 21399871]

97. Drebber U, et al. Altered levels of the onco-microRNA 21 and the tumor-supressor microRNAs 143 and 145 in advanced rectal cancer indicate successful neoadjuvant chemoradiotherapy. Int J Oncol. 2011; 39(2):409-415. [PubMed: 21567082]

98. Akcakaya $\mathrm{P}$, et al. miR-185 and miR-133b deregulation is associated with overall survival and metastasis in colorectal cancer. Int J Oncol. 2011; 39(2):311-318. [PubMed: 21573504]

99. Karaayvaz M, et al. Prognostic significance of miR-215 in colon cancer. Clin Colorectal Cancer. 2011; 10(4):340-347. [PubMed: 21752725]

100. Yu G, et al. Prognostic values of the miR-17-92 cluster and its paralogs in colon cancer. J Surg Oncol. 2011

101. Faber C, et al. Overexpression of Dicer predicts poor survival in colorectal cancer. Eur J Cancer. 2011; 47(9):1414-1419. [PubMed: 21345667]

102. Schetter AJ, et al. Association of Inflammation-Related and microRNA Gene Expression with Cancer-Specific Mortality of Colon Adenocarcinoma. Clin Cancer Res. 2009; 15(18):5878-5887. [PubMed: 19737943]

103. Rossi L, Bonmassar E, Faraoni I. Modification of miR gene expression pattern in human colon cancer cells following exposure to 5-fluorouracil in vitro. Pharmacol Res. 2007; 56(3):248-253. [PubMed: 17702597] 
104. Song B, et al. Mechanism of chemoresistance mediated by miR-140 in human osteosarcoma and colon cancer cells. Oncogene. 2009; 28(46):4065-4074. [PubMed: 19734943]

105. Song B, et al. Molecular mechanism of chemoresistance by miR-215 in osteosarcoma and colon cancer cells. Mol Cancer. 2010; 9:96. [PubMed: 20433742]

106. Mencia N, et al. Underexpression of miR-224 in methotrexate resistant human colon cancer cells. Biochem Pharmacol. 2011; 82(11):1572-1582. [PubMed: 21864507]

107. Chai H, et al. miR-20a targets BNIP2 and contributes chemotherapeutic resistance in colorectal adenocarcinoma SW480 and SW620 cell lines. Acta Biochim Biophys Sin (Shanghai). 2011; 43(3):217-225. [PubMed: 21242194]

108. Krutzfeldt J, et al. Silencing of microRNAs in vivo with 'antagomirs'. Nature. 2005; 438(7068): 685-689. [PubMed: 16258535]

109. Elmen J, et al. LNA-mediated microRNA silencing in non-human primates. Nature. 2008; 452(7189):896-899. [PubMed: 18368051]

110. Lanford RE, et al. Therapeutic silencing of microRNA-122 in primates with chronic hepatitis C virus infection. Science. 2010; 327(5962):198-201. [PubMed: 19965718]

111. Gumireddy K, et al. Small-molecule inhibitors of microrna miR-21 function. Angew Chem Int Ed Engl. 2008; 47(39):7482-7484. [PubMed: 18712719]

112. Young DD, et al. Small molecule modifiers of microRNA miR-122 function for the treatment of hepatitis C virus infection and hepatocellular carcinoma. J Am Chem Soc. 2010; 132(23):79767981. [PubMed: 20527935]

113. Ibrahim AF, et al. MicroRNA replacement therapy for miR-145 and miR-33a is efficacious in a model of colon carcinoma. Cancer Res. 2011; 71(15):5214-5224. [PubMed: 21690566] 


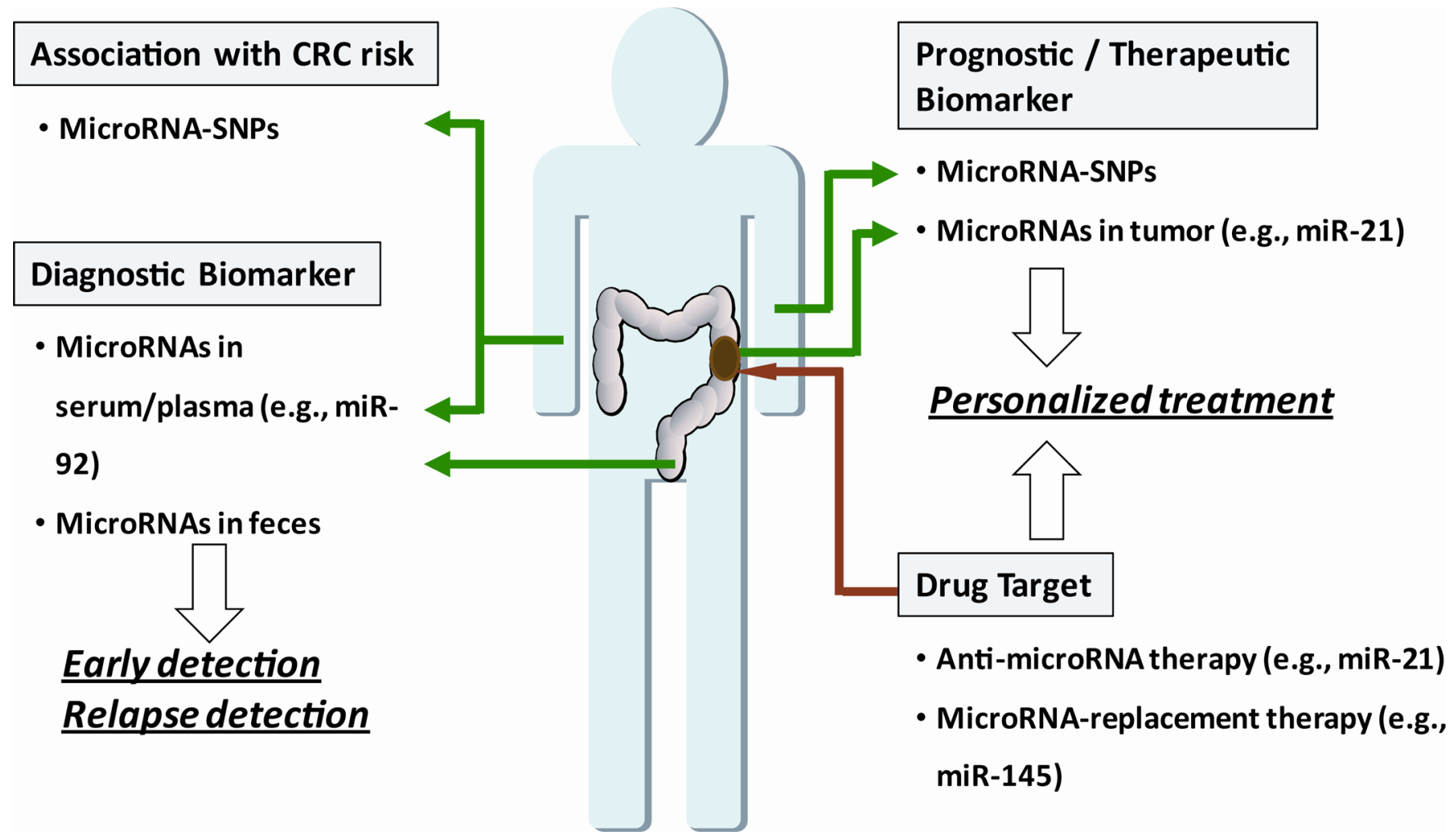

Figure 1.

Potential strategies for microRNA-based diagnostics and therapeutics for the detection and management of colon cancer. MicroRNA-related DNA polymorphisms may modify cancer risk. MicroRNAs detected in blood or feces may aid in the early detection of colon cancer. MicroRNA expression levels in tumors may help guide therapeutic decisions and have utility as a therapeutic target through microRNA inhibition or replacement strategies. 


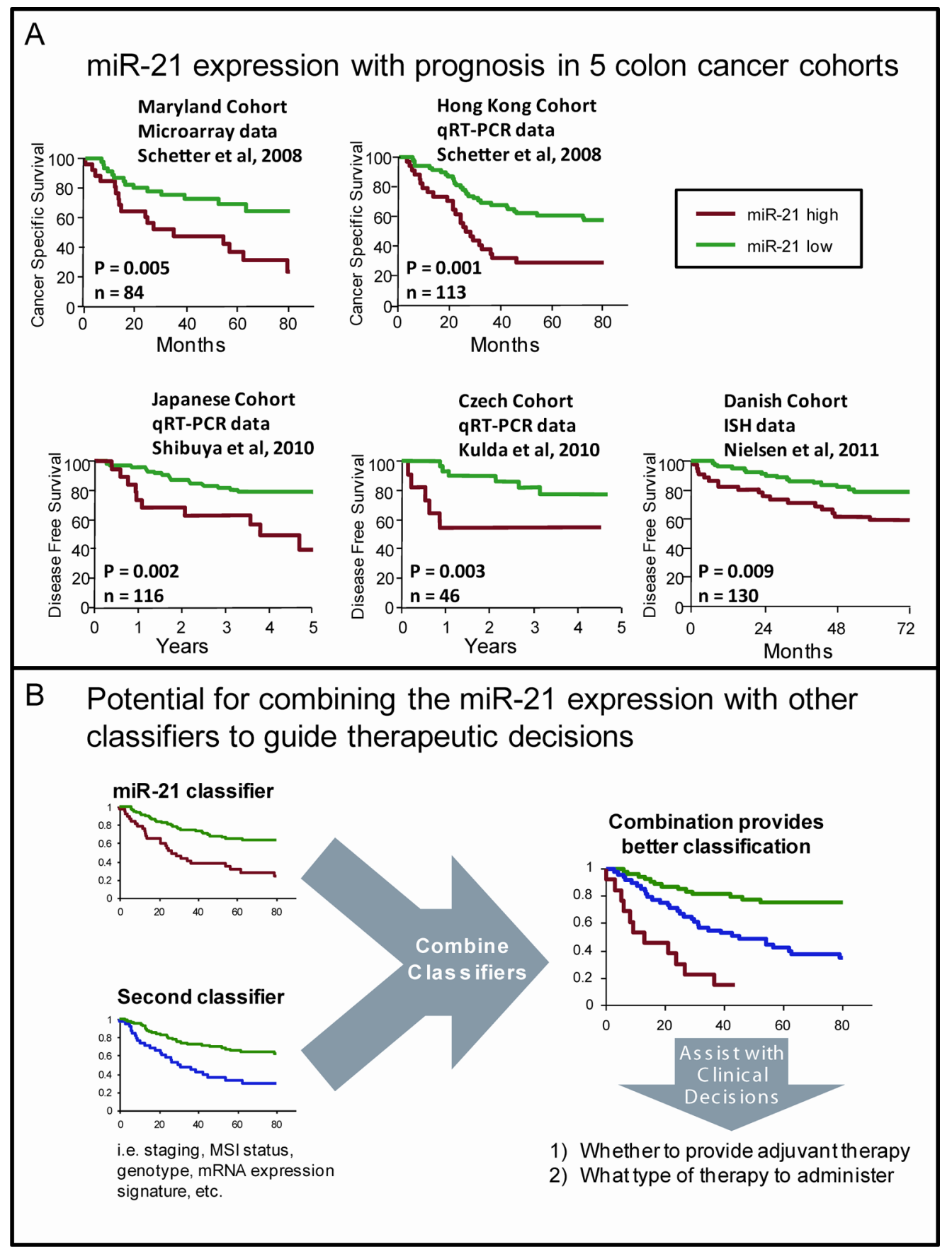

Figure 2.

MicroRNA-based prognostic and predictive classifiers for colon cancer. (A) High expression of $m i R-21$ is consistently associated with worse prognosis and therapeutic outcome in five colon cancer cohorts from four studies, suggesting miR-21 may have use as a prognostic or predictive classifier. (B) The combination of multiple, validated prognostic classifiers may provide improved risk stratification to help guide therapeutic decisions. ISH = in situ hybridization; qRT-PCR = quantitative reverse transcription polymerase chain reaction. 
Table 1

MicroRNAs with roles in colon cancer and their targets

\begin{tabular}{|c|c|c|}
\hline microRNA & $\begin{array}{l}\text { Tumor Suppressor or } \\
\text { Oncogene }\end{array}$ & $\begin{array}{c}\text { Examples of experimentally validated } \\
\text { microRNA Targets }\end{array}$ \\
\hline let-7 & Tumor Suppressor & $K R A S$ \\
\hline$m i R-17-92$ cluster & Oncogene & $E 2 F 1$ \\
\hline$m i R-21$ & Oncogene & $\begin{array}{c}\text { PDCD4, PTEN, RECK, NFIB, TPM1, SPRY2, } \\
\text { RHOB, TIMP3, maspin, CDC25a, TIAM1, } \\
\text { MSH2 }\end{array}$ \\
\hline $\operatorname{miR}-29$ & Tumor Suppressor & $M M P 2, D N M T 3 A / B$ \\
\hline miR-30a & Tumor Suppressor & $D T L$ \\
\hline $\operatorname{miR}-34 a$ & Tumor Suppressor & FRA1, SIRT1, MYC, BCL2 \\
\hline $\operatorname{miR}-95$ & Oncogene & $S N X 1$ \\
\hline $\operatorname{miR}-101$ & Tumor Suppressor & $\operatorname{COX} 2$ \\
\hline$m i R-135 a / b$ & Oncogene & $A P C$ \\
\hline $\operatorname{miR}-137$ & Tumor Suppressor & $C D C 42$ \\
\hline$m i R-143$ & Tumor Suppressor & $K R A S, D N M T 3 A, E R K 5$ \\
\hline$m i R-145$ & Tumor Suppressor & $\begin{array}{c}\text { IRS-1, c-Myc, YES1, STAT1, OCT4, SOX2, } \\
\text { KLF4,FLI1 }\end{array}$ \\
\hline $\operatorname{miR}-155$ & Oncogene & MLH1, MSH2, MSH6 \\
\hline$m i R-200 c$ & & $Z E B 1, Z E B 2$ \\
\hline $\operatorname{miR}-342$ & Tumor Suppressor & DNMT1 \\
\hline $\operatorname{miR}-365$ & Tumor Suppressor & $C C N D 1, B C L-2$ \\
\hline $\operatorname{miR}-451$ & Tumor Suppressor & $M I F$ \\
\hline $\operatorname{miR}-499$ & Oncogene & FOXO4, PDCD4 \\
\hline $\operatorname{miR}-675$ & Tumor Suppressor & $R B$ \\
\hline
\end{tabular}

